\title{
A HIERARCHICAL GECKO-INSPIRED SWITCHABLE ADHESIVE
}

\author{
Michael T. Northen \\ Materials Department \\ University of California at Santa Barbara \\ Santa Barbara, CA 93106, USA
}

\author{
Kimberly L. Turner \\ Mechanical Engineering Department \\ University of California at Santa Barbara \\ Santa Barbara, CA 93106, USA
}

\author{
Christian Greiner \& Eduard Arzt \\ Max Planck Institute for Metals Research, Heisenbergstrasse 3 \\ D-70569 Stuttgart, Germany
}

gecko to control adhesion through macroscopic movements of its toes. By flexing muscles the toe of gecko peels away from the surface in an inward fashion. This in itself aids in the detachment of the foot, just as peeling a piece of tape off of a surface is easier than trying to pull it off all at once. But there is more to the story. It has been shown theoretically (16) and experimentally (3) that the magnitude of adhesion of the setae is greatly dependent on the angle of applied pull-off force. Thus when the gecko goes from a squeezing mechanism to a peeling mechanism, the adhesive force goes from a maximum to a minimum (16). This can also be seen at the nanoscale as the resolved force on the spatulae changes the interactions from a frictional regime to a peeling regime, greatly reducing the adhesion (6). So through clever geometrical design of a hierarchical structure the gecko is able to control the adhesion mechanics from the macro-scale to nano-scale through a macroscopic muscle movement.

Previously, a synthetic system enhancing adhesion through a hierarchical structure was fabricated and tested(17). Just as the gecko uses this system to both increase and decrease adhesion, here a hierarchical structure has been fabricated to turn on and off adhesion. However, where the gecko uses muscle to actuate a $\beta$-keratin structure, the synthetic system uses a magnetic field to actuate nickel cantilevers. The nickel beams, when placed in a magnetic field, reorient themselves so that the terminal pad of the structure, responsible for adhesion, rotates orthogonally to an adhering surface. This conformational change effectively turns off the adhesion of the structure.

\section{Fabrication}

Released 150-500 nm thick and $100 \mu \mathrm{m}$ long nickel structures, coated with aligned vertical arrays of stiff polymeric nanorods $\sim 200 \mathrm{~nm}$ in diameter and $\sim 3 \mu \mathrm{m}$ tall, were fabricated using a combination of compatible massively parallel fabrication techniques. The fabrication process began by coating blank 4 inch (100) silicon wafers with a $1.4 \mu \mathrm{m}$ thick layer of image reversal photoresist, AZ 5214. The negative image of the desired platforms was then transferred into the resist across the entire wafer using a Karl Suss MA6 contact aligner. After developing, a $150 \mathrm{~nm}$ thick nickel layer was electron beam evaporated onto the entire wafer. The photoresist was then removed, via an ultrasonic acetone bath, lifting off the excess nickel. The wafer was then cleaned and dried and a $7 \mu \mathrm{m}$ layer of photoresist was spun onto the wafer surface, Shipley SPR 220-7. The positive pattern of the platforms was then transferred into the resist, aligned with the nickel platforms below. The resist and nickel pattern was then transferred into 
the exposed silicon alternating between a highly reactive mostly isotropic $\mathrm{SF}_{6}$ etch and a $\mathrm{C}_{4} \mathrm{~F}_{8}$ passivation deposition, the Bosch process, effectively etching vertically into the silicon. After etching approximately $30 \mu \mathrm{m}$ into the silicon, a sustained $\mathrm{SF}_{6}$ etch was performed to undercut the nickel/photoresist platforms. The released platforms were then placed in oxygen plasma with an applied bias between wafer and plasma, creating 200 nm diameter nanorods, orthogonally on the surface, with an aspect ratio of $\sim 15$, Fig. 1 .

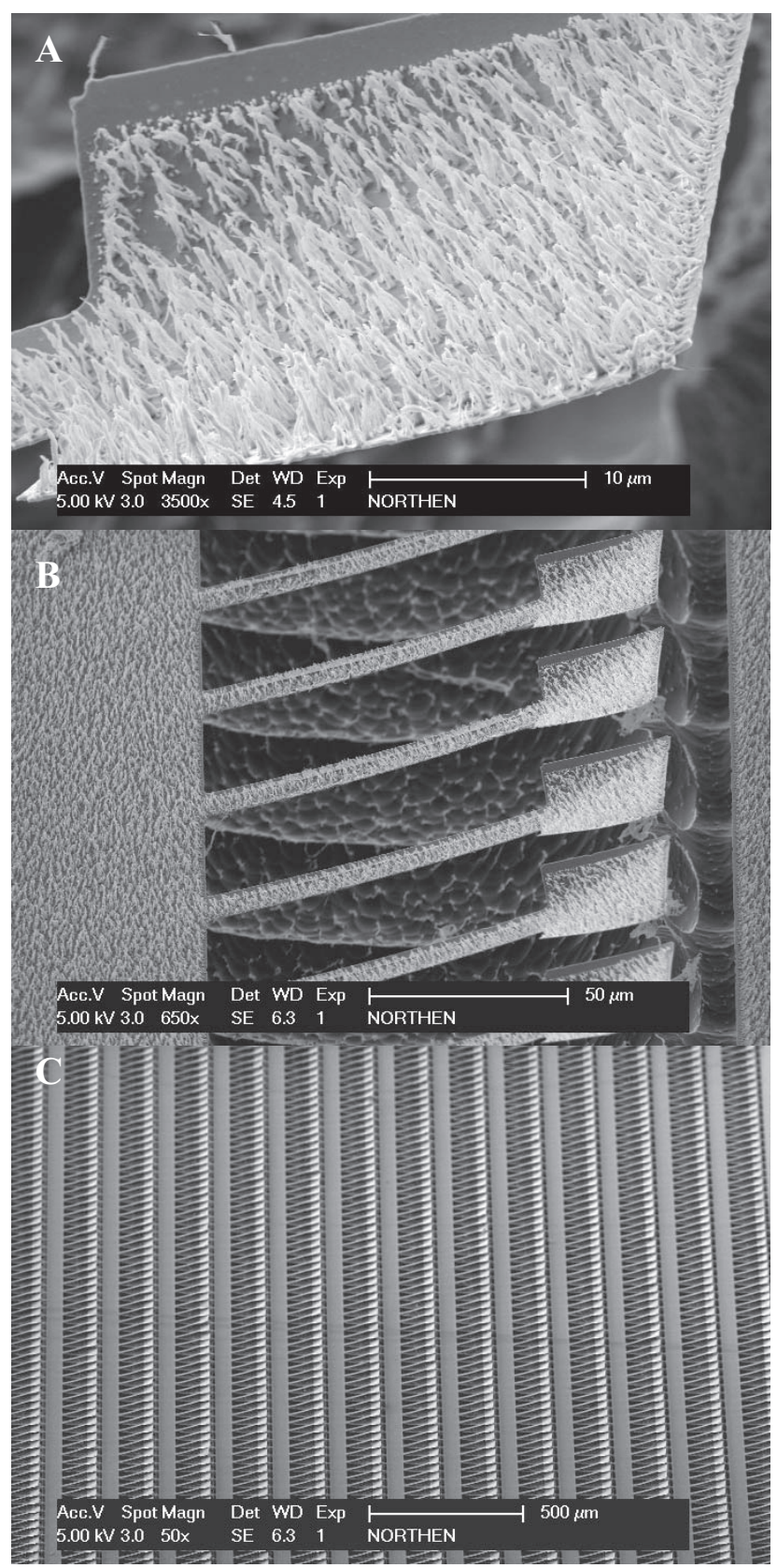

Fig. 1 - Electron micrographs of released structures. (A) Paddle surface coated with evenly spaced uncondensed aligned vertical polymer nanorods, scale bar $10 \mu \mathrm{m}$. (B) Freestanding nickel cantilevers and paddles coated with nanorods, scale bar $50 \mu \mathrm{m}$. (C) Lower magnification view of a portion of the array, scale bar $500 \mu \mathrm{m}$.

\section{Adhesion Testing}

The structures were characterized using a home built adhesion test apparatus, Fig. 2. The basic operating principle of the system is very similar to an atomic force microscope, except on a larger scale, where the deflection of a cantilever is monitored, using laser interferometry, to determine the forces applied to a probe tip. In this work, a custom $5 \mathrm{~mm}$ flat punch glass probe was manufactured. Proper registration of tip and stage was accomplished by the marriage of probe and cantilever (using high strength glue), with the cantilever secured in the apparatus, and the probe resting flush on the sample stage. Test samples were placed on the micropositioning stage and moved to near contact with the test probe. The probe was then lowered using a piezo electric actuator, and proper probe/sample alignment was observed through a horizontally oriented stereomicroscope. Actuation of the probe and data collection was performed using an automated National Instruments Lab View program. The program simultaneously moved the probe towards the surface and monitored the deflection of the cantilever. Through calibration of the cantilever, spring constant equal to $137.1 \mathrm{~N} / \mathrm{m}$, it was possible to determine the interaction forces between the flat punch probe and the test surface. Upon withdrawal from the surface, adhesion would produce a characteristic pull-off event, evident in the negative dip of the force curve, Fig. 4. The reversible adhesive was tested with and without a Neodymium Iron Boron rare earth metal magnet below the silicon chip.

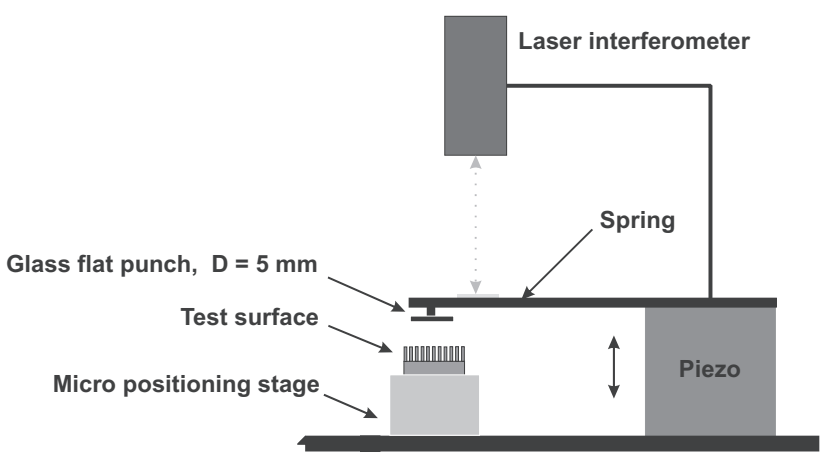

Fig. 2 - Schematic of the adhesion test apparatus. A laser interferometer monitors the deflection of a glass cantilever spring as a piezo actuator moves a $5 \mathrm{~mm}$ glass flat punch into and away from the test surface. The interaction forces are calculated by relating the stiffness and deflection of the cantilever upon contact with the surface. 
Fabrication of the multi-scale structures required the integration of two different processing modalities. The nickel platform microstructures were photolithographically defined and etched using standard microfabrication reactive ion etching. The vertically aligned polymeric nanostructures were created through a stochastic growth method. Both methods employ batch fabrication techniques and scalable for production quantities. So in addition to being the first actuated hierarchical biomimetic adhesive, this system does not present a technological dead end.

The bilayer of the photoresist coating the nickel beams induces a stress mismatch which causes the beams to bend up and out of the device plane, Fig. 1. The upwards bent cantilevers aid in adhesion through enhanced structural compliance, and move the active portion of the adhesive away from the surface. With the active portion of the adhesive isolated the properties of the adhesive could then be controlled by actuating the platforms. High-aspect-ratio ferromagnetic structures have been shown to rotate within a magnetic field to align their long axis with a magnetic field (18). When the structures were placed on top of a permanent magnet the platforms were observed to rotate about their long axis, Fig. 3. This rotation is attributed to the preferential alignment of the long axis of the width of the pad in the magnetic field. This rotation reduces the orthogonal contact area, as evident in the stereo-micrograph in figure 3.
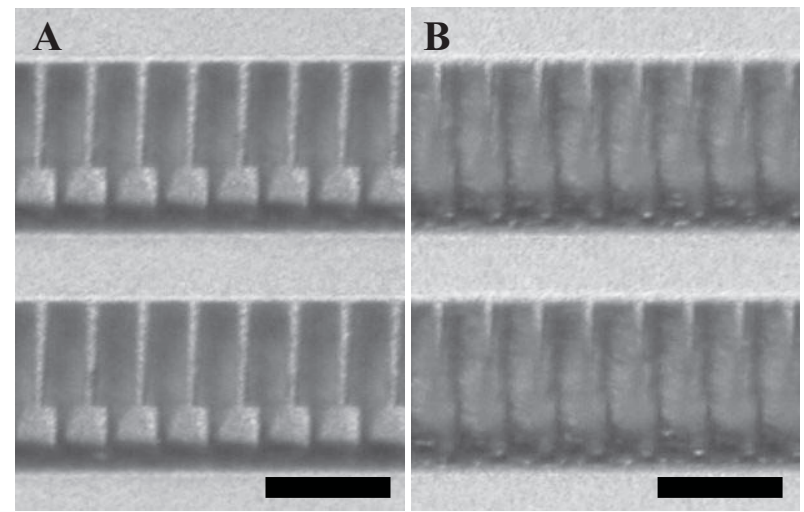

Fig. 3 - Stereomicrographs of the adhesive: (A) in the ' $O N$ ' state, no applied magnetic field, with the adhesive paddles facing vertically; and (B) in the 'OFF' state, with an applied magnetic field rotating the paddles sideways, concealing the adhesive faces. Scale bars, $100 \mu \mathrm{m}$.

Adhesion testing of the structures, without an applied magnetic field, produced loading and unloading curves with a characteristic pull-off event, Fig. 4. The magnitude of the pulloff force was taken to be the adhesion, and was observed to vary with the maximum applied normal load (due to slight misalignments between the flat punch and the test surface) until a saturation adhesion strength of $\sim 17 \mathrm{~Pa}$ was observed (obtained by dividing the adhesion force by the projected area of the pad surfaces), Fig. 4.
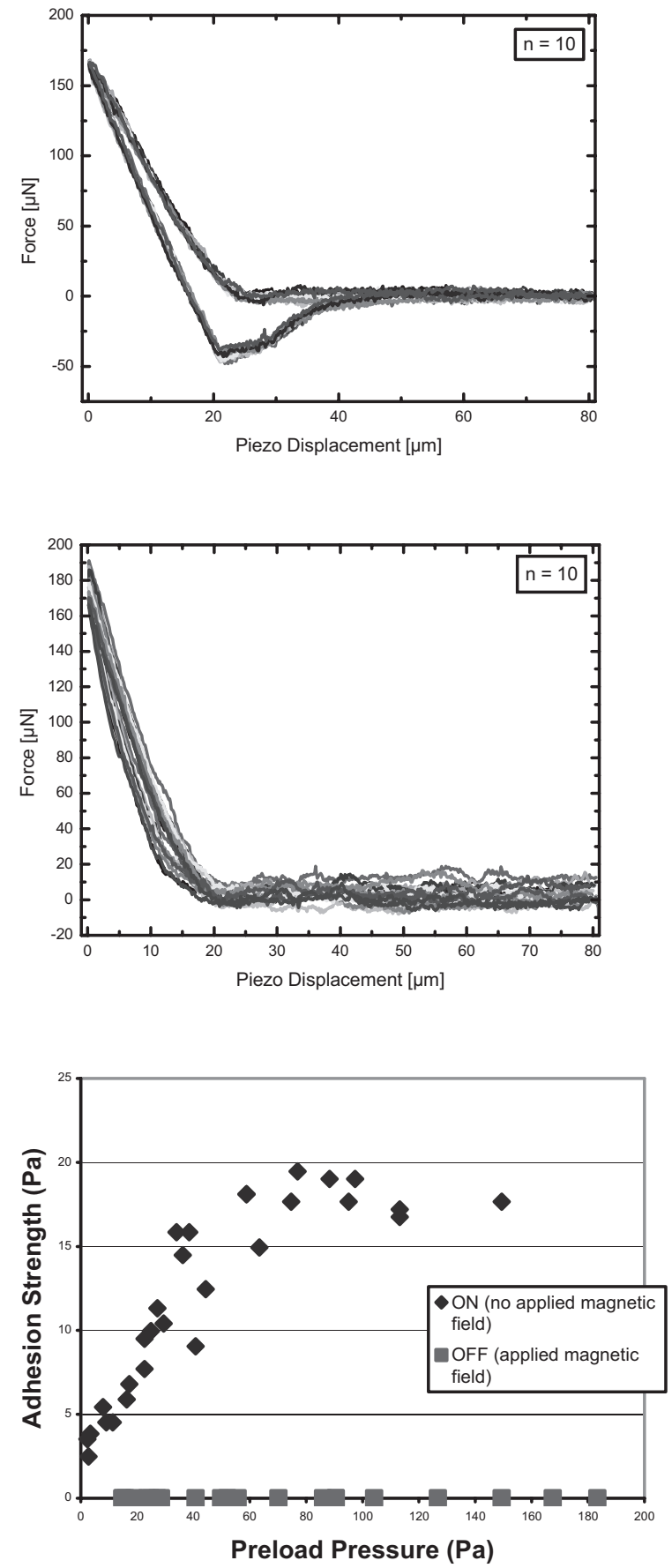

Fig. 4 - Adhesion results showing the on/off behavior of the structures without and with an applied magnetic field, respectively. The plots represent loading and unloading curves during testing, where in the ' $O N$ ' state distinctive pull-off events were observed (top) and in the 'OFF' state no pull-off events were observed (bottom). Strength values were obtained by dividing the interaction force by the contact area of the paddles. In the ' $O N$ ' state, the devices showed an initial increase in adhesion with preload force, characteristic of increased surface contact with applied load. In the off state there was no detectable adhesion. 
Alignment issues, surface inconsistencies and unknown probe geometries have presented difficulties in quantification of non-pressure-sensitive-adhesives. One suggested metric is to simply divide the adhesion force by the maximum preload force, $\mu^{\prime}=\mathrm{F}_{\text {adhesion }} / \mathrm{F}_{\text {normal }}$, thus distinguishing these systems from pressure sensitive adhesives (19). In the system described here the maximum $\mu$ ' value was found to be $\sim 1.5$, occurring at the minimum detectable level of adhesion (limited by the noise level of the instrumentation). This value offers a substantial increase from previous synthetic work; where Northen and Turner reported a $\mu^{\prime}=0.125$ (17), and Geim et al. a $\mu^{\prime}=0.06(20)$, but still falls short of the gecko with $\mu^{\prime}=8$ to 16 (19).

In contrast to the adhesion seen in a rest state, the application of magnetic field to the structures produced a catastrophic loss of adhesion, Fig. 4. For no tests performed was any adhesive event observed. This complete reduction in adhesion is attributed to the concealing of the nanorod coated platforms from the test probe. Under an applied magnetic field the platforms tend to rotate about the long axis of the cantilever. This rotation leaves the edge of the platforms facing in the normal direction and the sticky face to the side - turning a cheek to adhesion, if you will.

Additionally, an increase in surface stiffness was seen with an applied magnetic field. The twisting of the cantilevers increases the second moment of area of the structures, relative to the indenting tip, increasing the stiffness and consequently reducing the compliance of the system. Although not a primary factor in the reduction of adhesion, reduction of the compliance of the system serves to lock out one of the hierarchies of this multi-scale adhesive. Ultimately the sideways rotated paddles will contact the underlying substrate and statically inhibit contact between the adhesive and the test substrate, completely turning off the adhesive.

\section{Conclusion}

In this paper a novel approach has been presented for micro- and nano-fabricating a synthetic analogue to the gecko adhesive system. The hierarchical system is composed of aligned vertical nanorods coating flexible micron scale cantilever paddles. The paddles, composed of nickel, rotate when subjected to a magnetic field. This rotation conceals the nanostructures on the paddle surface and greatly reduces the available surface area for adhesion. Testing of the system showed reversible adhesion behavior switching from a $\mu$ ' value $\left(\mathrm{F}_{\text {adhesion }} / \mathrm{F}_{\text {preload }}\right)$ of $\sim 1.5$ (largest reported value for a biomimetic system to date (19)) to a value less than the noise level in the instrumentation. Thus an active hierarchical structure, inspired by the gecko, has been fabricated and demonstrated to display controlled and reversible adhesion.

\section{References}

1. Aristotle, Historia animalium translated by Thompson, $D^{\prime} A . W$. (Oxford, The Clarendon Press, 350 B.C.E. (1918)), pp. 584.

2. K. Autumn et al., Nature 405, 681 (2000).

3. K. Autumn et al., Proc. Natl. Acad. Sci. USA 99, 12252 (September 17, 2002, 2002).

4. G. Huber et al., PNAS 102, 16293 (2005).

5. W. Sun, P. Neuzil, T. S. Kustandi, S. Oh, V. D. Samper, Biophys. J. 89, L14 (2005).

6. B. N. J. Persson, S. Gorb, Journal of Chemical Physics 119, 11437 (2003).
7. C. Y. Hui, N. J. Glassmaker, T. Tang, A. Jagota, Journal of the Royal Society London Interface 1, 35 (2004).

8. E. Arzt, S. Gorb, R. Spolenak, Proc. Natl. Acad. Sci USA 100, 10603 (2003).

9. M. Scherge, S. Gorb, Biological Micro- and Nanotribology: Nature's Solutions (Springer, Berlin, 2001), pp.

10. R. Ruibal, V. Ernst, Journal of Morphology 117, 271 (1965).

11. U. Hiller, Z. Morph. Tiere 62, 307 (1968).

12. A. P. Russell, Journal of Zoology London 176, 437 (1975).

13. E. E. Williams, J. A. Peterson, Science 215, 1509 (1982).

14. H. J. Gao, X. Wang, H. M. Yao, S. Gorb, E. Arzt, Mechanics of Materials 37, 275 (Feb-Mar, 2005).

15. R. Spolenak, S. Gorb, E. Arzt, Acta BIOMATERIALIA 1, 5 (2005).

16. H. Gao, H. Yao, Proc. Nat. Acad. Sci. U. S. A. 101, 7851 (2004).

17. M. T. Northen, K. L. Turner, Nanotechnology 16, 1159 (2005).

18. J. Judy, R. Muller, H. H. Zappe, Journal of Microelectromechanical Systems 4, 162 (1995).

19. K. Autumn, in Biological Adhesives A. S. a. J. Callow, Ed. (Springer Verlag, 2006).

20. A. K. Geim, S. V. Dubonos, I. V. Grigorieva, K. S. Novoselov, A. A. Zhukov, Nature Materials 2, 461 (2003). 